\title{
An Atypical Site of Vascular Tumor: IVPG of Right Renal Vein
}

Paolo Umari, Giovanni Liguori, Rossana Bussani, Michele Bertolotto and Carlo Trombetta

Department of Urology, University of Trieste, Strada di Fiume 447, 34149 Trieste, Italy

\section{Introduction}

Intravenous pyogenic granuloma (IVPG) is an extremely rare polypoid variant of lobular capillary hemangioma [1]. It was first described in 1979 by Cooper et al. and a most recent review found less than 30 cases reported in literature until now [2-4]. IVPG has been seen in patients from 15 to 66 years of age (average 38 years) and with a slight female predominance [5]. These lesions are commonly thought to arise in upper extremities and in neck and head veins [6,7]. IVPG is a benign intravascular lesion with no potential for haematogenous spread [8]. The proliferation is entirely confined within a vein lumen and a fibrovascular stalk attaches the lesion to the wall of the vein with a nearby small artery thought to provide its blood supply [2]. The surface of the tumor exposed to the vein lumen is covered by an endothelial lining. Typical histological features include lobules of capillaries within an edematous fibromyxoid stroma [3]. Histological appearances are similar to those of a classical pyogenic granuoma, but with absence of inflammatory changes [2]. The treatment of choice is complete local excision and the prognosis is excellent [9]. There are no known associated risk factors and the pathogenesis of the lesion has not been determined [10].

\section{Case Report}

A hypertensive, but otherwise healthy 50 -year-old man presented to
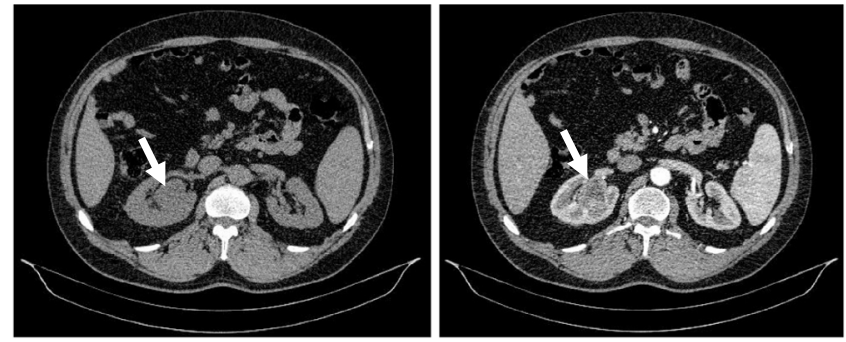

Figure 1: CT scan revealed the presence of a $4 \mathrm{~cm}$ mesorenal mass in the right kidney (left) with a significant enhance after administration of the contrast agent (right). A thin plane of cleavage between the nodule and the ventral bifurcation of the renal artery can be identified.
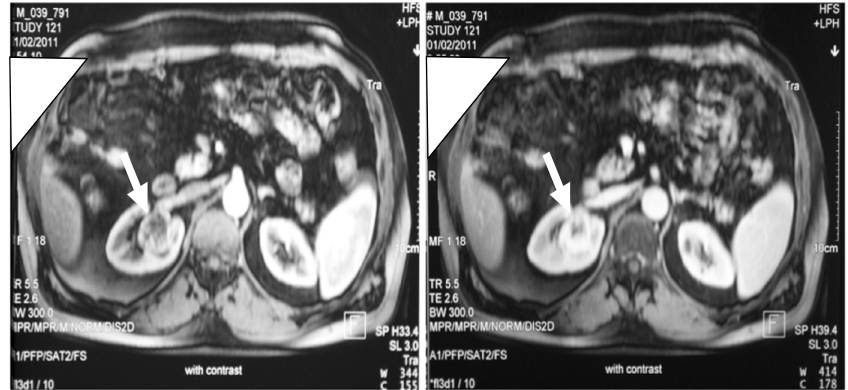

Figure 2: MRI before (left) and after (right) intravenous administration of gadolinium. our Department with the diagnosis of renal cancer. His medical history and laboratory data were unremarkable. The patient denied symptoms such as pain, weight loss and changes in nutrition in the recent past. His physical examination was unremarkable, with no lymphadenopathy and abdominal or lumbar masses. The tumor was seen for the first time during an abdominal ultrasonography performed to investigate lower urinary tract symptoms related to a benign prostate hyperplasia. Afterwards the patient underwent further imaging studies; the CT scan revealed the presence of a $4 \mathrm{~cm}$ right meso-renal enhancing mass, highly suggestive of malignancy (Figure 1). The mass was located in close proximity to the renal vessels, preventing the surgical enucleation. The magnetic resonance imaging confirmed the presence of a mass in the right kidney with a better contrast resolution (Figure 2). Moreover before surgery he underwent a contrast enhanced ultrasound, which showed perfusion and reperfusion after micro-bubble breakage (Figure 3).

The patient was seen also by other specialists from different departments and the indication for surgery was a common opinion. $\mathrm{He}$ subsequently underwent a right radical nephrectomy; in the operating room a right subcostal incision was made and the ureter was completely freed, ligated and dissected. The right renal vein and artery were identified and ligated. After that the entire right kidney was mobilized and removed with the preservation of the adrenal gland.

At examination, the mass appeared solid, spherical, pale brown, well demarcated and adherent to the vein wall by a fibrovascular stalk. No necrosis and hemorrhage were identified. It measured $3 \times 3 \mathrm{~cm}$, $2 \times 2 \mathrm{~cm}$ (Figure 4). The tumor consisted of proliferation of small sized capillary vessels separated by fine grey septs (Figure 5). Microscopically it was composed of lobules of capillaries within an edematous fibromyxoidstroma (Figure 6). Vessels were lined by endothelium and a stroma containing smooth muscle fibers. The endothelial cells showed a marked nuclear atypia and mitotic figures and a slight degree of nuclear enlargement. The surface of the tumor exposed to the vein lumen was also lined by endothelial cells.

Immunotyping of the surgical specimen showed strong expression for the following markers: CD34, CD31, alpha-smooth muscle actin and desmin (Figures 7 and 8). These findings were in accordance with a diagnosis of IVPG.

*Corresponding author: Paolo Umari, Department of Urology, University of Trieste, Strada di Fiume 447, 34149 Trieste, Italy, Tel: +393313321600; E-mail: paoloumari@alice.it

Received November 13, 2013; Accepted November 25, 2013; Published November 27, 2013

Citation: Umari P, Liguori G, Bussani R, Bertolotto M, Trombetta C (2013) An Atypical Site of Vascular Tumor: IVPG of Right Renal Vein. J Vasc Med Surg 1: 120 doi: 10.4172/2329-6925.1000120

Copyright: ( 2013 Umari P, et al. This is an open-access article distributed under the terms of the Creative Commons Attribution License, which permits unrestricted use, distribution, and reproduction in any medium, provided the original author and source are credited. 


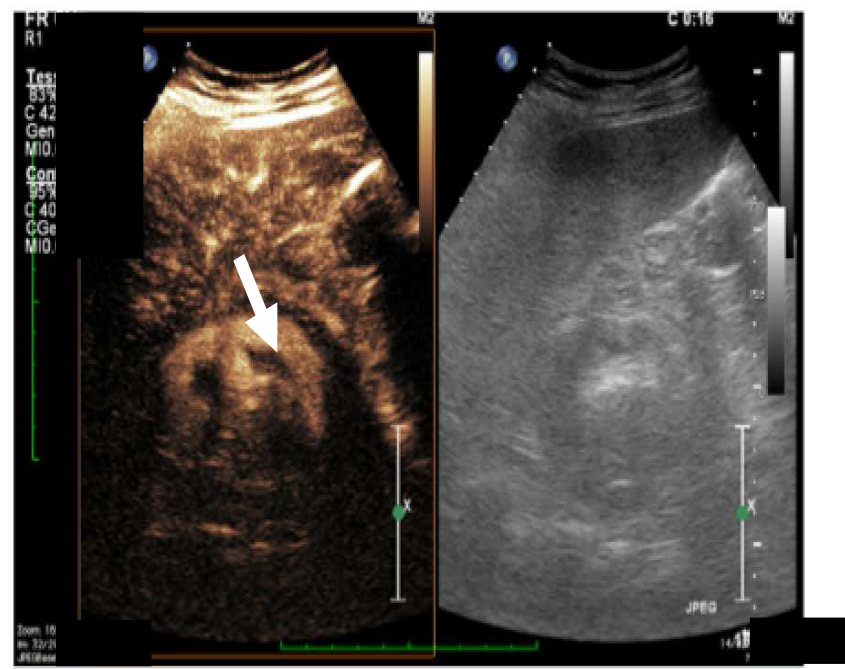

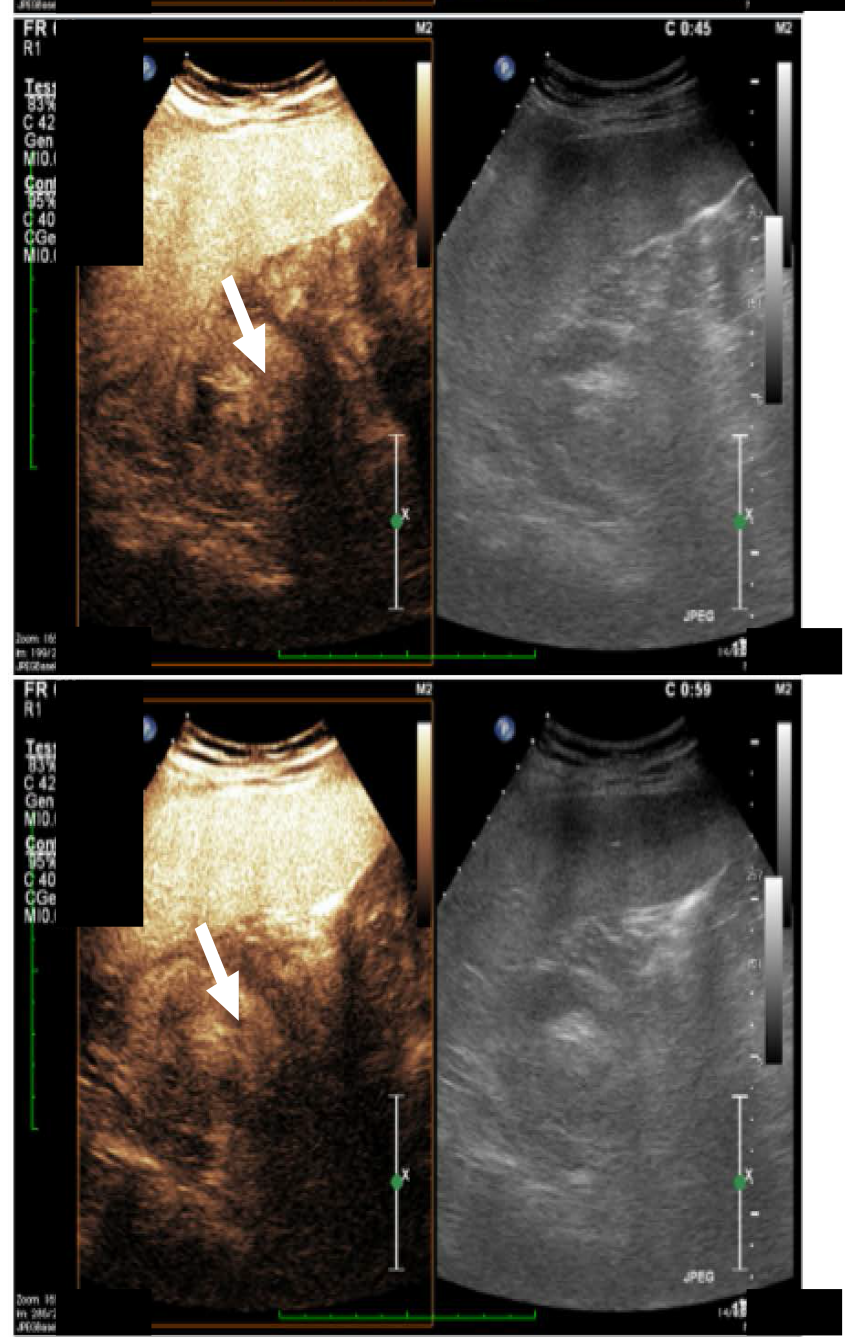

Figure 3: Contrast enhanced sonography; strong enhancement at $16 \mathrm{sec}$ $45 \mathrm{sec}$ and $60 \mathrm{sec}$ (from top to bottom).

The postoperative course of our patient was free of complications and he had a fast recovery. After 9 months follow-up he didn't show signs of disease and imaging revealed no recurrence.

\section{Discussion}

Early-stage tumors in asymptomatic patients are increasingly discovered as a result of the easy availability and widespread use of ultrasonography. In our patient an abdominal ultrasound was performed to investigate lower urinary tract symptoms related to benign prostate hyperplasia. A mass was discovered and it was found to be a very rare tumor.

IVPG is a benign entity that has important clinical implications; in fact it can easily mimic a malignant lesion at imaging [2]. The clinical picture of the IVPG is highly nonspecific and insufficient to make a precise diagnosis $[3,5,11]$. There are no known associated risk factors and the nature of the lesion remains obscure [10]. However, the histological picture of IVPG is particularly characteristic and manifests a remarkable resemblance throughout series described until now [3]. Immunostaining of the surgical specimen is considered an useful aid for diagnosis $[2,10]$. Our tumor showed a strong expression for antigens as CD34, CD31 alpha-smooth muscle actin and desmin that helped us to put the correct diagnosis. The tumor should be differentiated from other intravascular lesions including thrombus, angiosarcoma, hemangiothelioma and intravascular papillary endothelial hyperplasia. Angiosarcoma does not show a distinctive lobular pattern but rather is usually poorly organized, diffuse and infiltrative; the cytologic pattern is characterized by nuclear hyperchromasia and pleomorphisms. The hemangiothelioma has an intermediate position between well differentiated hemangioma and anaplastic angiosarcoma. Finally intravascular papillary endothelial hyperplasia can be distinguished from intravenous pyogenic granuloma by its papillary architecture, presence of hemosiderin deposits and association with organized thrombi $[2,5,6]$.

The prognosis of IVPG is usually excellent [2]. However, the growth rate of this tumor is unknown and it could continue to grow or recur if not completely resected [2]. In our case aradical nephrectomy was indicated, because of the tumor position in close proximity to the main renal vessels.

\section{Conclusion}

Intravenous pyogenic granuloma (IVPG) can easily make us to suspect a malignant disease. The surgeon is confronted with serious difficulties in diagnostic and therapeutic choices. Imaging can help us to define the lesion, but histopathological examination is necessary to make the definitive diagnosis. Even if we have very sophisticated diagnostic tools available nowadays, sometimes exist a really large gap between imaging and reality; in fact the treatment of the urological patient with the diagnosis of renal tumor, requires a strong collaboration between surgeon, radiologist and pathologist. The prognosis of complete resected IVPG has proved to be excellent, so we think that in our case the patient will not have recurrences.

\section{References}

1. Johnson NA, Haeney J, Yii NW (2011) Intravenous pyogenic granuloma of the finger. J Hand Surg Eur Vol 36: 251-252.

2. Pradhan S, Bazan H, Salem R, Gusberg RJ (2008) Intravenous lobular capillary hemangioma originating in the iliac veins: a case report. J Vasc Surg 47: 1346-1349.

3. Cooper PH, McAllister HA, Helwig EB (1979) Intravenous pyogenic granuloma A study of 18 cases. Am J Surg Pathol 3: 221-228.

4. Takeuchi M, Hara S, Itoh T (2012) A case of intravenous lobular capillary hemangioma of the renal vein mimicking renal cell carcinoma. Pathol Int 62 441-443. 
Citation: Umari P, Liguori G, Bussani R, Bertolotto M, Trombetta C (2013) An Atypical Site of Vascular Tumor: IVPG of Right Renal Vein. J Vasc Med Surg 1: 120 doi: $10.4172 / 2329-6925.1000120$

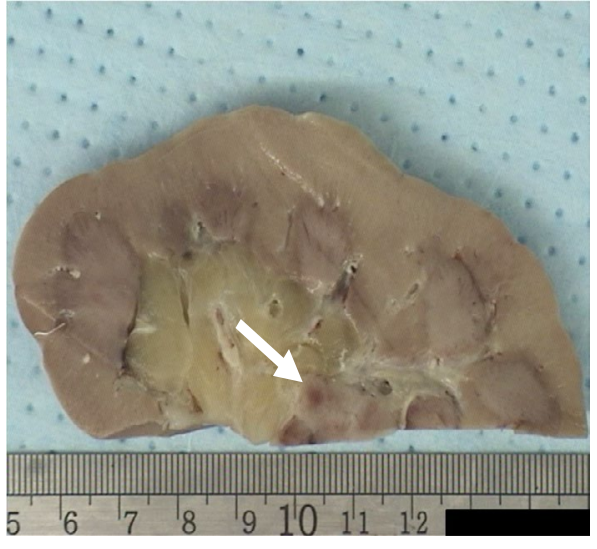

Figure 4: The arrow shows the exact site of the mass.

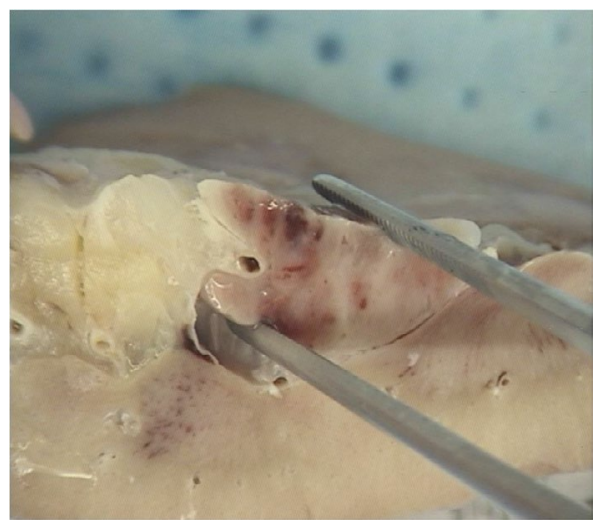

Figure 5: Photo of a macrocopic section of the tumor..

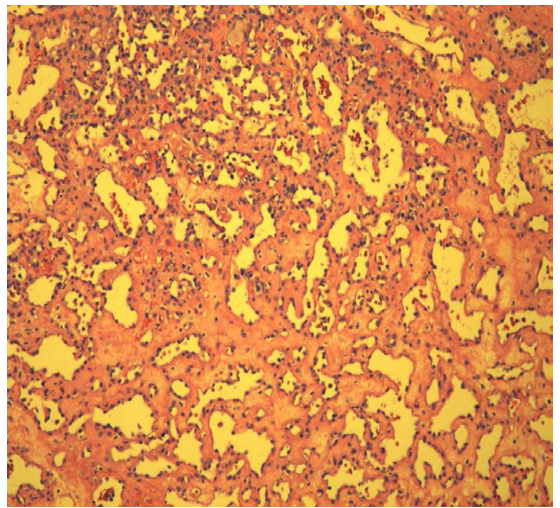

Figure 6: Hematoxylin-eosin stain of the tumor; the basic structural unit is the lobular arrangement of capillaries in an edematous fibromyxoidstroma, magnification $10 x$.

5. Kamishima T, Hasegawa A, Kubota KC, Oizumi N, Iwasaki N, et al. (2009) Intravenous pyogenic granuloma of the finger. Jpn J Radiol 27: 328-332.

6. Ghekiere O, Galant C, Vande Berg B (2005) Intravenous pyogenic granuloma or intravenous lobular capillary hemangioma. Skeletal Radiol 34: 343-346.

7. Saad RW, Sau P, Mulvaney MP, James WD (1993) Intravenous pyogenic granuloma. Int J Dermatol 32: 130-132.

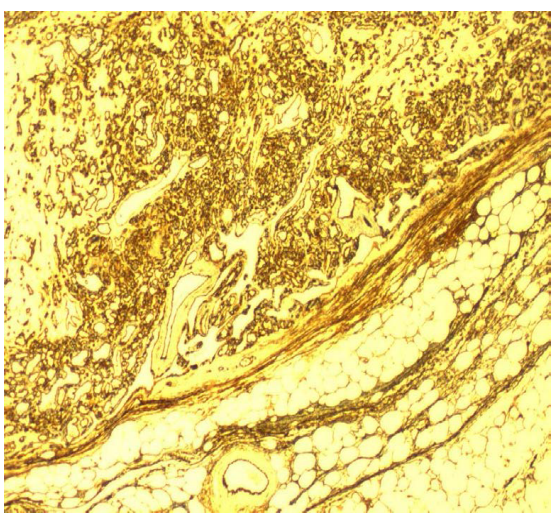

Figure 7: Immunohistochemistry stain demonstrates positiveness for CD34 a fibrovascular stalk attaches the lesion to the wall of the vein magnification $2.5 \mathrm{x}$.

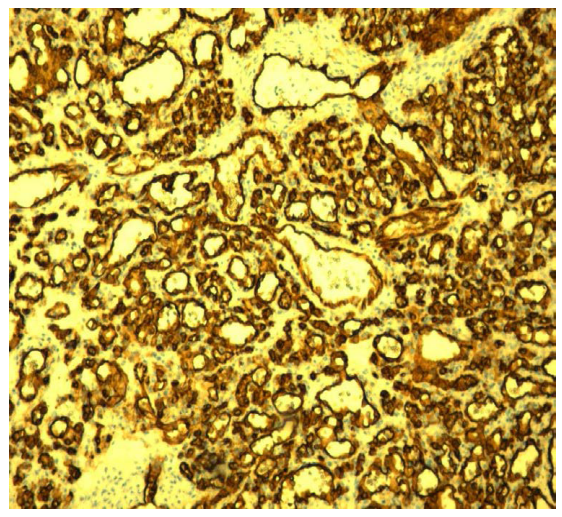

Figure 8: Positive immunohistochemical profile of the surgical specimen for CD34, magnification 10x

8. Anderson WJ (1985) Intravenous pyogenic granuloma of the hand. J Hand Surg Am 10: 728-729.

9. DiFazio F, Mogan J (1989) Intravenous pyogenic granuloma of the hand. J Hand Surg Am 14: 310-312.

10. Qian LH, Hui YZ (2001) Intravenous pyogenic granuloma: immunohistochemical consideration--a case report. Vasc Surg 35: 315-319.

11. Song MG, Kim HJ, Lee ES (2001) Intravenous pyogenic granuloma. Int J Dermatol 40: 57-59. 